\title{
Diffraction Amplitude for Fractures Imaging \& Hydrocarbon Prediction
}

\author{
Yasir Bashir $^{1,2}$, Deva Prasad Ghosh ${ }^{1}$, Chow Weng Sum ${ }^{1}$ \\ ${ }^{I}$ Centre of Excellence in Subsurface Seismic Imaging \& Hydrocarbon Prediction (CSI), \\ ${ }^{I}$ Department of Petroleum Geoscience, Universiti Teknologi PETRONAS, Malaysia
}

\begin{abstract}
Seismic diffractions are often considered as noise and have been suppressed during processing intentionally or implicitly. Diffraction hyperbola actually have the information of discontinuity such as fault, fracture, Karst and edge of salt flanks, which always contain valuable information for geological interpretation and reservoir modeling. These diffractions play an important role to illuminate the small scale events which are crucial for structural traps such as fractured reservoir. Another important part of diffraction is to improve the amplitude recovery at the certain point through diffraction summation. These diffracted waves have been recognized as physically reliable carriers of high and even super-resolution structural information. This research work illustrates the different behavior of diffraction hyperbola comparing with different velocities and depths. Phase change in amplitude in $180^{\circ}$ from +ive to -ive and the decay in amplitudes directly proportional to the angle of incident. Synthetic data set is used for the analysis of amplitude preservation through diffraction imaging. Outcome of the research demonstrate the consideration of diffraction imaging for discontinuities, complex structure and small scale reservoir imaging, which subsidize a good impact to search the hydrocarbon potential reserves. Another aspect is cover that describes the importance of migration aperture selection for integration of the diffractions on the basis of frequency of the data, as high frequency will produce less diffraction response then low frequency. Last but not least, the diffraction imaging improves the spatial resolution of individual faults and fracture below the resolution of reflections.
\end{abstract}

Key Words: Seismic Imaging, Diffractions amplitude, Frequency, Aperture, Velocity

\section{Introduction}

Seismic diffraction events are produced because of important small scale elements in the subsurface, such as faults, fractures, channels, and rough edges of salt bodies, or small changes in the seismic reflectivity, such as those produced by fluid occurrence or fluid movement for the period of production. These diffracted waves are those that comprise the most appreciated information (Fomel, Landa, and Taner 2007). The diffraction theory sometimes makes it impossible to understand the qualitative properties of diffraction phenomena(E Landa 2010). Further, these diffractions data are also used for traveltime approximation for anisotropic imaging (Waheed, Alkhalifah, and Stovas 2013).

Diffraction hyperbolic patterns occur frequently in seismic sections, and their existence is usually taken as evidence of abrupt discontinuities in the subsurface reflector geometry(Hilterman 1975). A geophysicist knows about the ray path geometry, which at every "point" diffractor, edge of reflector, and fault give rise to a hyperbolic pattern on a zero offset section(Berryhill 1977). Most fault planes are not vertical, they are inclined. A series of diffractions that originate along a given fault surface are translated horizontally so that the apex of each curve is on the fault plane.

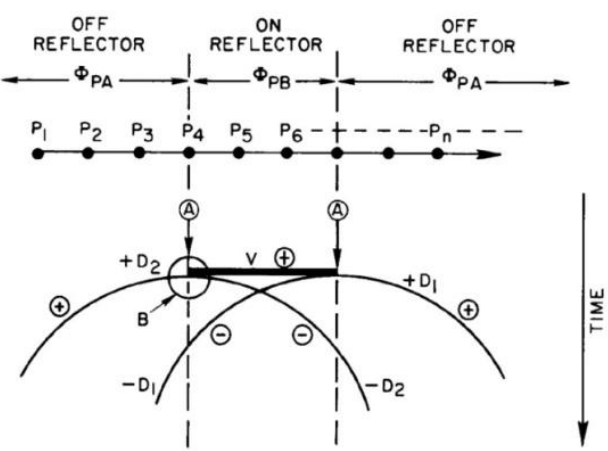

Fig. 1.Explanation of the diffraction hyperbola, generated at the edge of the reflectors (D1 and D2). Phase change of 180 degrees on either side of the diffracting edge (+ and -) (Trorey 1970). 
Diffracted and reflected seismic waves are fundamentally different physical phenomena (KlemMusatov et al. 1994, Moser and Howard 2008). As shown in Figure 1, the diffraction hyperbola is produced at the edge of the horizontal reflector; it is because of the impendence contrast. Further diffraction hyperbolas have +ive to -ive amplitudes which generally decrease with time. The regular practice during processing is that reflected wave are tuned, and diffracted waves are suppressed by being considered as noise, but these diffracted events are responsible for carrying the most important information about the subsurface.

The understanding of the separation seismic diffraction is not innovative. (Harlan, Claerbout, and Rocca 1984) used diffraction to estimate the velocity from forward modelling and the local slant stack. (Evgeny Landa and Keydar 1998) used a common-diffraction-point section for imaging of diffraction energy and detecting local heterogeneities; and (Söllner and Yang 2002)simulate diffraction responses for enhancing velocity analysis.

This paper illustrate the practical implementation of diffraction theory and increase the understanding of diffraction hyperbola due to faulted structure and bring the possibility to interpret the true structure. In which diffraction hyperbolas are divided into two parts, in which one is the algebraic signs of amplitudes phase change of 180 degree and other is that apex of hyperbola have maximum energy, while the part below the reflection have same energy (Hilterman 1970). We distinguish the reflective and diffractive event to the wave propagation and recognize the diffractive components as a key ingredient to image the true subsurface structure. We present the double square root equation that is fundamental of diffraction theory and practicing the theory in real earth model example.

\subsection{Diffraction Modelling}

\section{Methodology}

Principle of diffraction theory is established on the basis of that, the subsurface behaves like an acoustic media, it means we do not consider the share wave, only the P-waves are consider for diffraction, with constant velocity and low reflectivity. The scalar wave equation is used for satisfying wave equation.

- Reflector is a collaborative of distinct elements.

- The response is a sum of or integration of each element.

- Zero offset solution.

When the source and receiver are separated, the envelope of arrival times (diffraction curve) has a different shape due to the increased travel times.

"Double Square Root Equation (DSR)" formula for travel time is:

$$
T_{L}=\sqrt{\left(\frac{T m}{2}\right)^{2}+\left(\frac{L-\frac{x}{2}}{V}\right)^{2}}+\sqrt{\left(\frac{T m}{2}\right)^{2}+\left(\frac{L+\frac{x}{2}}{V}\right)^{2}}
$$

where $\mathrm{Tm}$ is the time, $\mathrm{V}$ is velocity, $\mathrm{x}$ is separation between the normal incident (single radical equation) and shot receiver separate (double square root equation). Curve is greatest at the observation location over the point source(Ghosh. 2012). At location L varnished and expression above collapse, so equation can be written as

$$
T L=\sqrt{T m^{2}+\frac{L^{2}}{V^{2}}}
$$

This is the usual expression for reflection times form a horizontal reflector. This observation forms the basis of velocity determination method. Using equation (2) on MATLAB code, we observe the behaviour or diffraction hyperbola by taking different constant velocity and keep increasing randomly, as shown in Fig (2-4).

\subsection{Amplitude Interpretation:}

Understanding of diffraction amplitude helps for the interpretation at pinchouts and discontinuities. Figure 2 a is a graphical representation of the diffraction which is cause by the edge of the reflector in case of shot point is away from the diffractor point. Which can be described by the relation below

$$
\text { Diffraction Effect }(D . E)=\left(\frac{Z}{R_{0}}\right)^{2} \frac{\text { Shaded Area } S_{f p}}{\text { Circular Area } S_{r p}}
$$

Where the subscript fp refers to the plane terminated by the fault, which is referred to the fault plane and rp refers to the reference plane (Hilterman 1975). 
Figure $2 \mathrm{~b}$ is a representation of the diffraction effect when the shot point is directly over the fault location. In this case, half of the energy is reflected and half passes by the diffraction plane. This effect can be measured by above equation as D.E=0.5. Amplitude is the very crucial aspect of the imaging which is a way forward for hydrocarbon prediction through amplitude interpretation. Figure 3 is the description of amplitude decay in the zero source receiver distance that is produce by the implementation of the equation by Barry hill modification(Berryhill 1977)

$$
D_{0}\left(t^{\prime}\right)=\frac{\operatorname{Cos} \theta_{0}}{\pi} \frac{t_{0}^{2}}{\left(t^{\prime}+t_{0}\right)^{2}}
$$

Where $t^{\prime}=t^{\prime}+t_{0}$ is time measured after the onset time $t_{0}$, as shown in figure $3, \theta$ is the angle measured between the normal to the reflecting plane and the minimum-time ray-path to the edge of the plane. Figure 3 is drawn to emphasize that the amplitude decay with the angle of incident wave, starting form 10 degree to 90 degree.
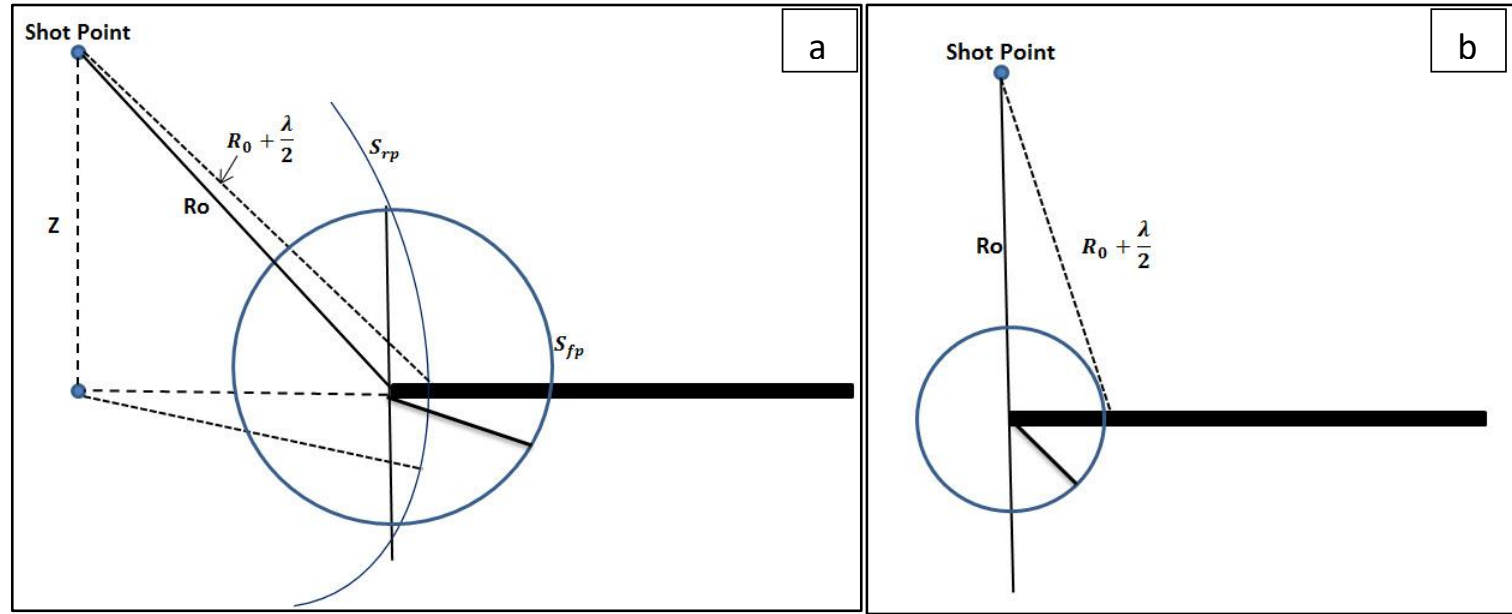

Fig 2: Graphical solution for the diffraction consequence from a plane edges. (a) When the source is not exactly above the diffracted point. (b) Shot is exactly above the diffracted point.

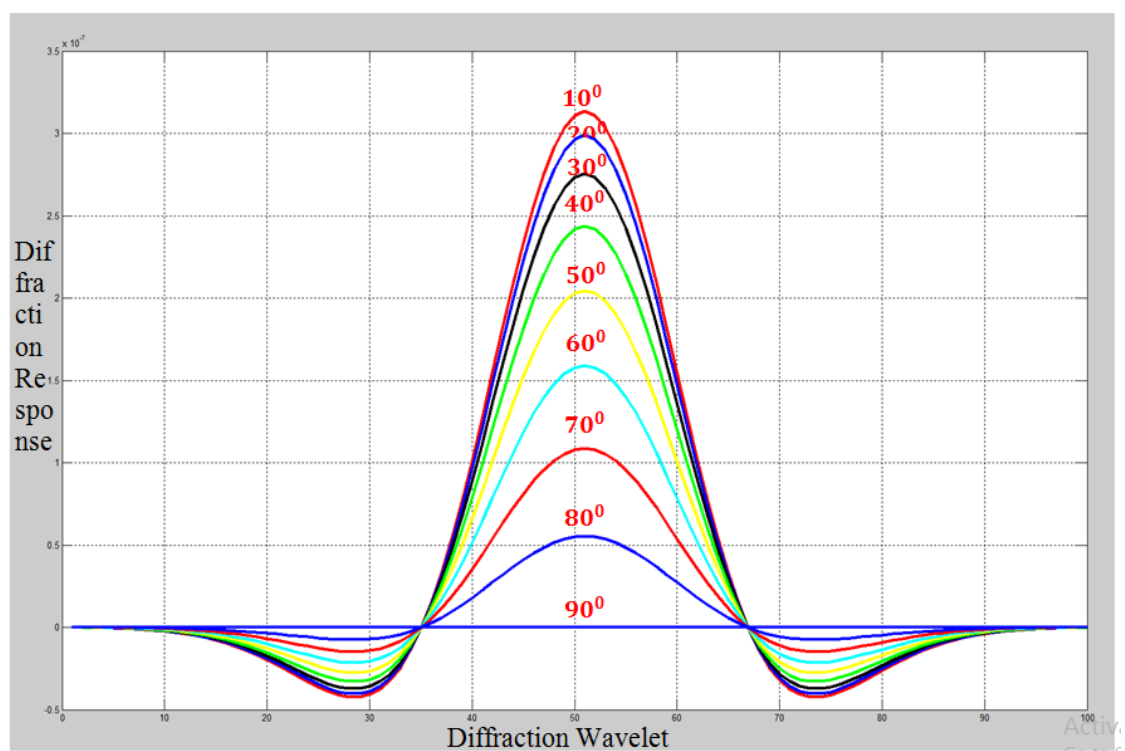

Fig 3: Diffraction amplitude decay for the zero shot receiver distance case. As the amplitude of the wavelet is decrease as angle of incident increase.

It needs to be highlighted that in the zero source receiver distance geometry, the above equation is rather restrictive, in the sense that diffraction amplitude are predicted to die off quite rapidly as the magnitude of angle $\theta_{0}$ increase from zero. 


\section{Numerical Implementation}

Three constant velocity numerical models and two variable velocity models are presented to illustrate the diffraction hyperbolic behaviour with difference velocity and depth of the diffracted point. Figure 4, 5 and 6 show constant velocity 2000, 3500 and $5000 \mathrm{~m} / \mathrm{sec}$ simultaneously, offset is on x-axis and time on y-axis, four diffraction hyperbolas are shown at each interval of $1 \mathrm{sec}$. Annotations from the figures below, point toward that the diffraction hyperbola is being spread out as time/depth increases. Velocity is also one of the major components for diffraction hyperbolic behaviour. By comparing Figure 4 and Figure 6, on Y-axis, scale is same but we can clearly see the difference that at a higher velocity, hyperbola spreads out more than lower velocity.

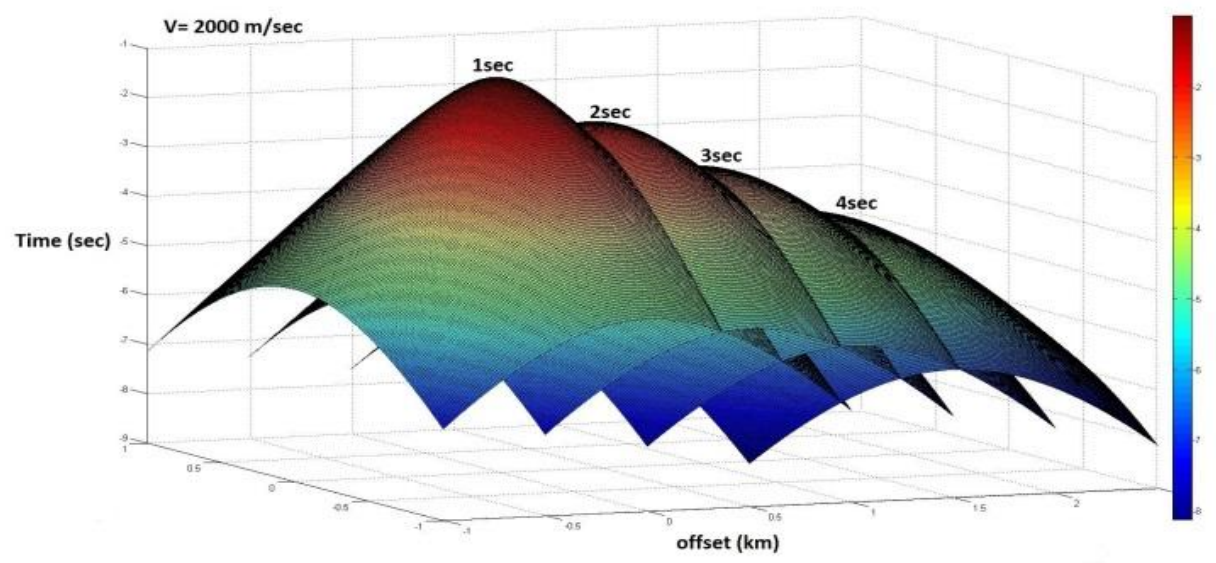

Fig.4. Diffraction curve of velocity $2000 \mathrm{~m} / \mathrm{sec}$ shows, if velocity is constant but the time is increasing, that will effect on hyperbola, as time increase curvature of hyperbola will spread out.

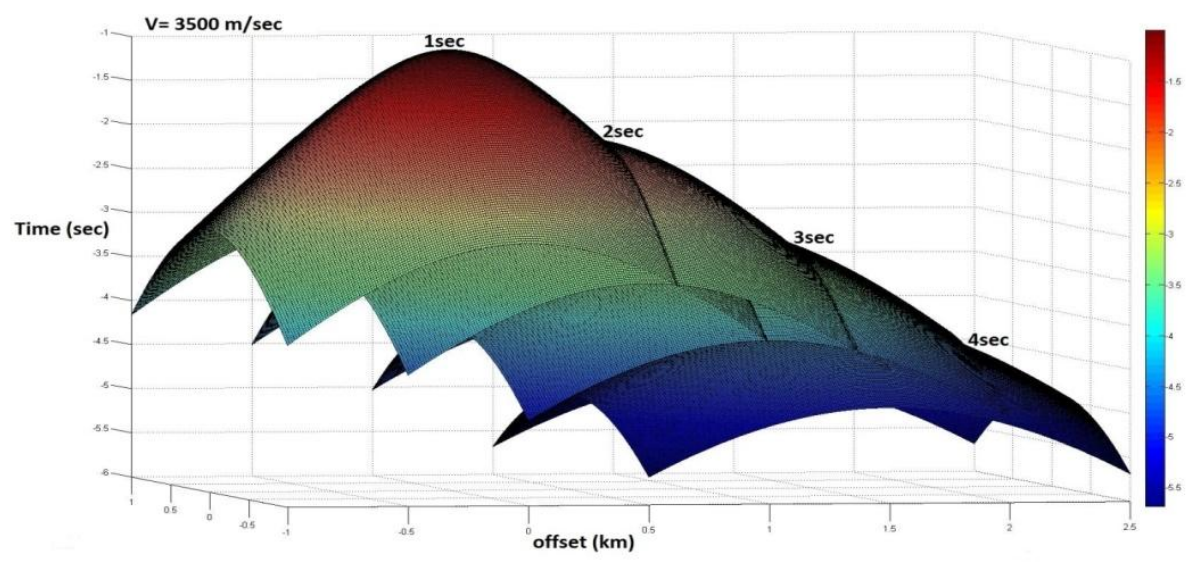

Fig.5. Diffraction curve of velocity $3500 \mathrm{~m} / \mathrm{sec}$ shows, constant velocity but higher than Figure 1, so curvature of hyperbola spread out more than low velocity.

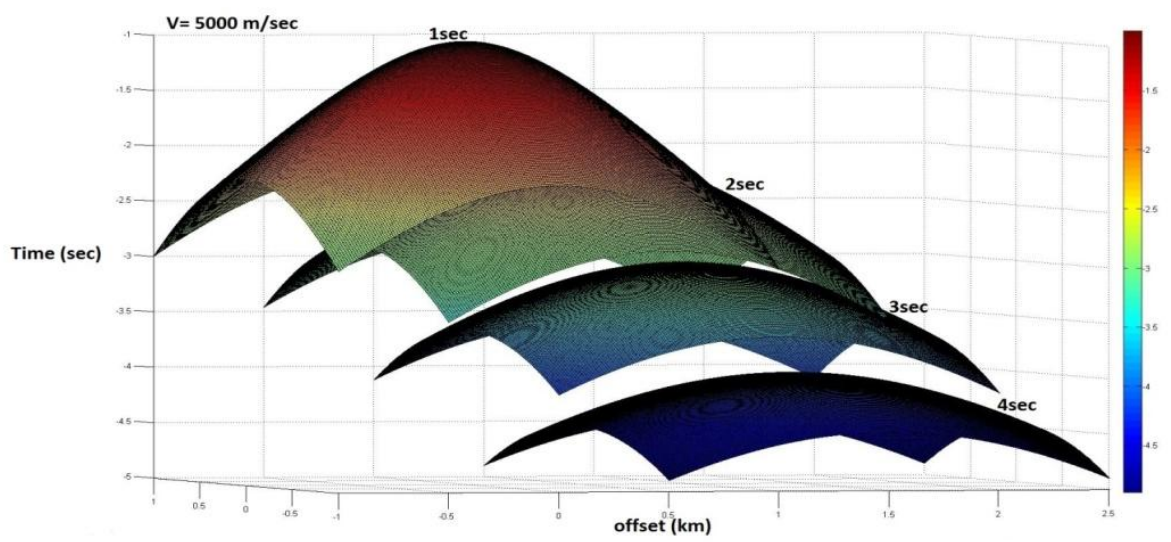

Fig.6. Diffraction curve of velocity $5000 \mathrm{~m} / \mathrm{sec}$ shows constant but higher velocity than figure 1 and 2 , therefore diffraction hyperbolic curvature is spread out more in higher velocity with same time. 
Now, in figure 7 we consider increasing velocity with time increase. Figure 7 illustrate increasing velocity with time as in a real earth model structure. So, the diffraction hyperbola spread out more with increasing depth. Figure 8 shows decreasing velocity with time, which describe if we have high velocity layer above and low velocity layers bellow. In this case we can observe the diffraction hyperbola have less curvature but in lower layer curvature will increase more.

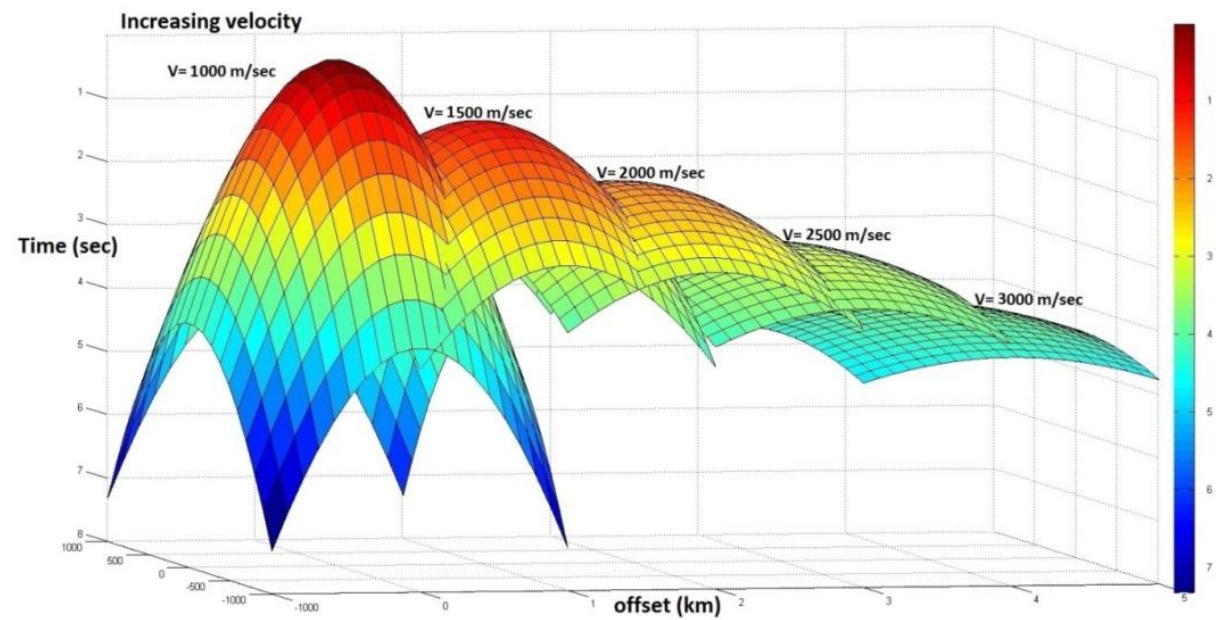

Fig.7. Diffraction curves at increasing velocity.

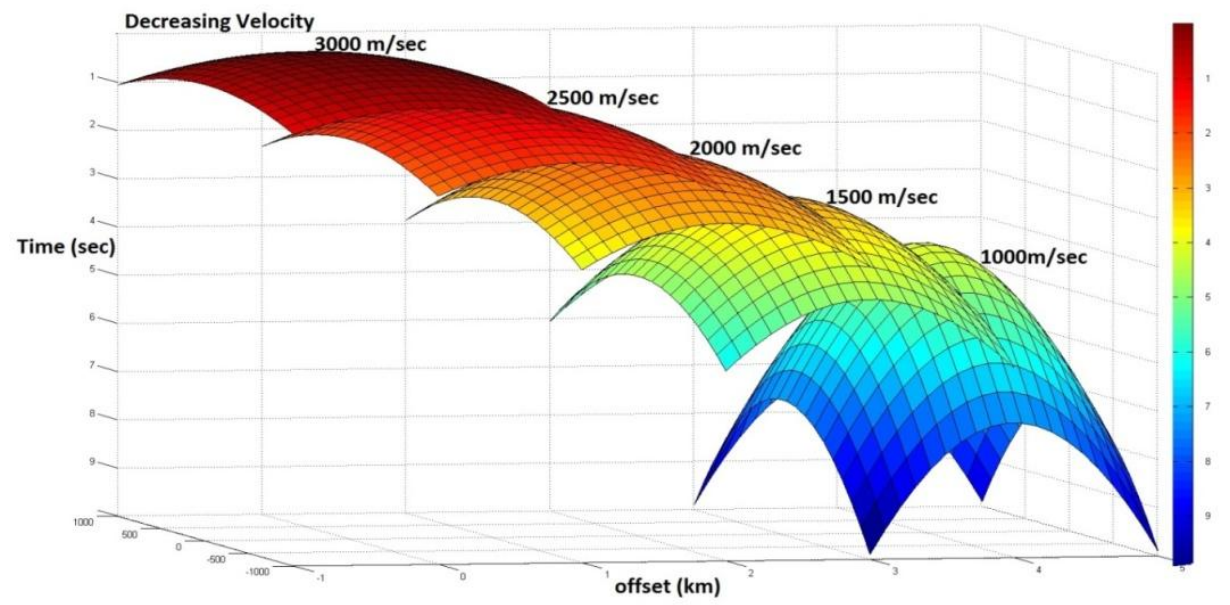

Fig. 8. Diffraction curve at decreasing velocity

\section{Results \& Discussions}

The primary goal of imaging and diffraction imaging in particular, is to obtain images of subsurface and structural interpretation for hydrocarbon prediction. Pre-processing of the seismic data, for structural imaging or reservoir property estimation, almost always include a stacking of pre-stack gathers, and hence a kinematical correction of offset-dependent move-out, with the aim of increasing the signal-to-noise ratio. There are numerous motivations to study and imaging of diffractions in the depth domain. The look of the diffractions in the seismic data is frequently indication for intensely complexities and a strong inhomogeneous trend model. Such events with complex properties can be invalidating the assumption of the time migration, so that pre-stack depth imaging is formerly the method of choice. Another way to be success of identifying and isolating diffractions depends on the quality of focusing or the accurateness of the velocity model. If the velocity model is exact and detailed enough for an optimally focused full-wave depth image, then for the diffraction image no additional focusing is needed. Forward modelling is perform for calculating synthetic seismic zero-offset data, the finite-difference wave equation approximation is used which is shown in Figure 9-10. Above situation under consideration is shown in figure 7. Pairs of 41 source and receiver are located at a distance of $20 \mathrm{~m}$ and depth is $2000 \mathrm{~m}$. A reflecting plane terminates along a steeply dipping fault edge and produces diffraction hyperbola at the edge of reflector, so the interpreter can find the abrupt change from diffraction and mark the fault point.

Figure 9-10 are similar model but we consider the distance of intruded layer (hole in reflector model). Figure 9 shows 100 meter intruded layer that produce the seismic diffraction because of intrusion edges. If we do not consider diffraction and suppress during processing we will just observe that it is a straight horizontal 
reflector. Until we keep increasing the distance of intruded layer till 300 meter (Figure 10) but that does not shows the discontinuity in reflectivity unless we consider diffraction hyperbola in to account for processing the data. To illustrate the idea and comprehensive effect of diffraction imaging we consider Model-1 as 100 meter hole (Figure 9) and Modle-2 as 300 meter hole in reflector (Figure 10). Two types of modelling is perform on these models with the dominant frequency of $30 \mathrm{~Hz}$ and $80 \mathrm{~Hz}$ to illustrate the effect amplitude preservation at the fault point in the imaged section with the optimum aperture size. In this manner, the low frequency data was imaged on a larger size of aperture than the high frequency data as shown in Figures 11 to 14. Figures 11 and 12 illustrate the low frequency data on Model-1 and Model-2, respectively, which had $100 \mathrm{~m}$ and $300 \mathrm{~m}$ hole widths. Figure 11a and Figure 12a show the un-Image zero-offset seismic gather, 11b and 12b are after the application of the Kirchhoff migration using the aperture size of $100 \mathrm{~m}$; but, we can see the diffractions are not stack properly because of choosing the small aperture. For sections $11 \mathrm{~b}$ and $12 \mathrm{~b}$, the sharp edges are not illuminated until we increased the migration aperture until $400 \mathrm{~m}$ as shown in Figures $11 \mathrm{~d}$ and $12 \mathrm{~d}$. Here, if we consider the real earth model, generally the velocity increases with the depth, and the frequencies affected by earth materials, so the high frequencies of the containing wave will be absorbed. In deep sections we have only low frequencies; because of these low frequencies a high diffraction responses will be produced. In actuality, we have to use a larger aperture for a deep diffraction hyperbolic summation. For our model, we did not have that depth, so we used a small aperture that was enough to image the diffractions. Figures 13 and 14 illustrate the high frequency synthetic seismic gather on Model-1 and Model-2, the hole width was $100 \mathrm{~m}$ and $300 \mathrm{~m}$, respectively. Figure 13a and Figure 14a are zero-offset un-Image seismic gathers in which we cannot identify the faults or hole in reflector, which is indicated by the blue colour arrow. $13 \mathrm{~b}$ and $13 \mathrm{c}$ are after implementation of the Kirchhoff migration using the aperture size of $100 \mathrm{~m} .13 \mathrm{c}$ and $14 \mathrm{c}$ are also after the migration using aperture length of $300 \mathrm{~m}$. This example shows that utilizing and selection of right aperture for the data set to preserve the amplitude of the diffractions. We have demonstrated that, the higher frequency data set, the smaller migration aperture is enough for the integration of all amplitudes form the flanks of the diffraction hyperbola. High frequencies data is good for the vertical resolution such as thin bed imaging but horizontal resolution can be increase by considering diffractions in our processing.
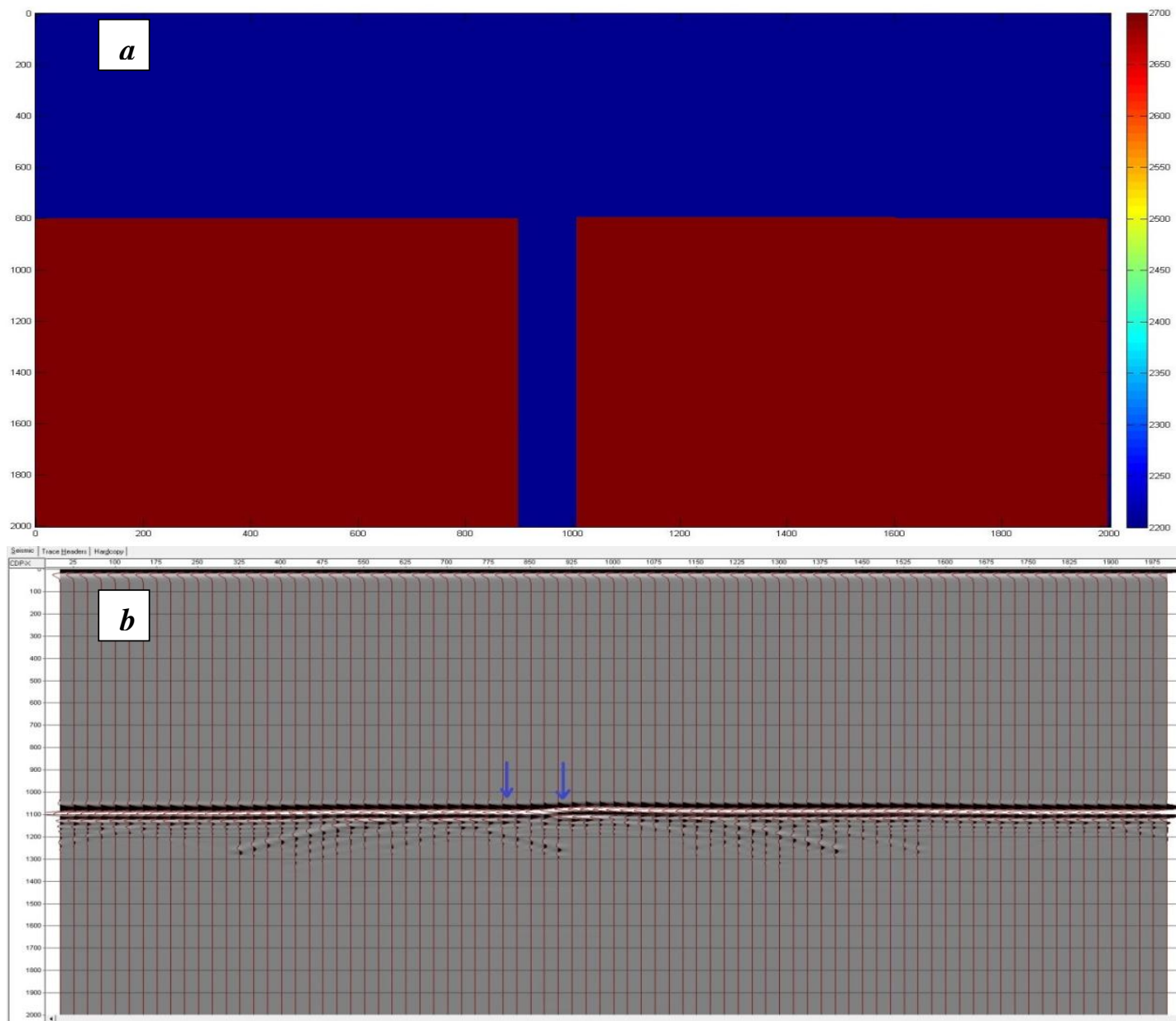

Fig.9: (a) Hole in reflector model with two layer having different velocity $1500 \mathrm{~m} / \mathrm{sec}$ and $3000 \mathrm{~m} / \mathrm{sec}$ and intruded layer width is $100 \mathrm{~m}$ and (b) Zero-offset synthetic seismic gather. 

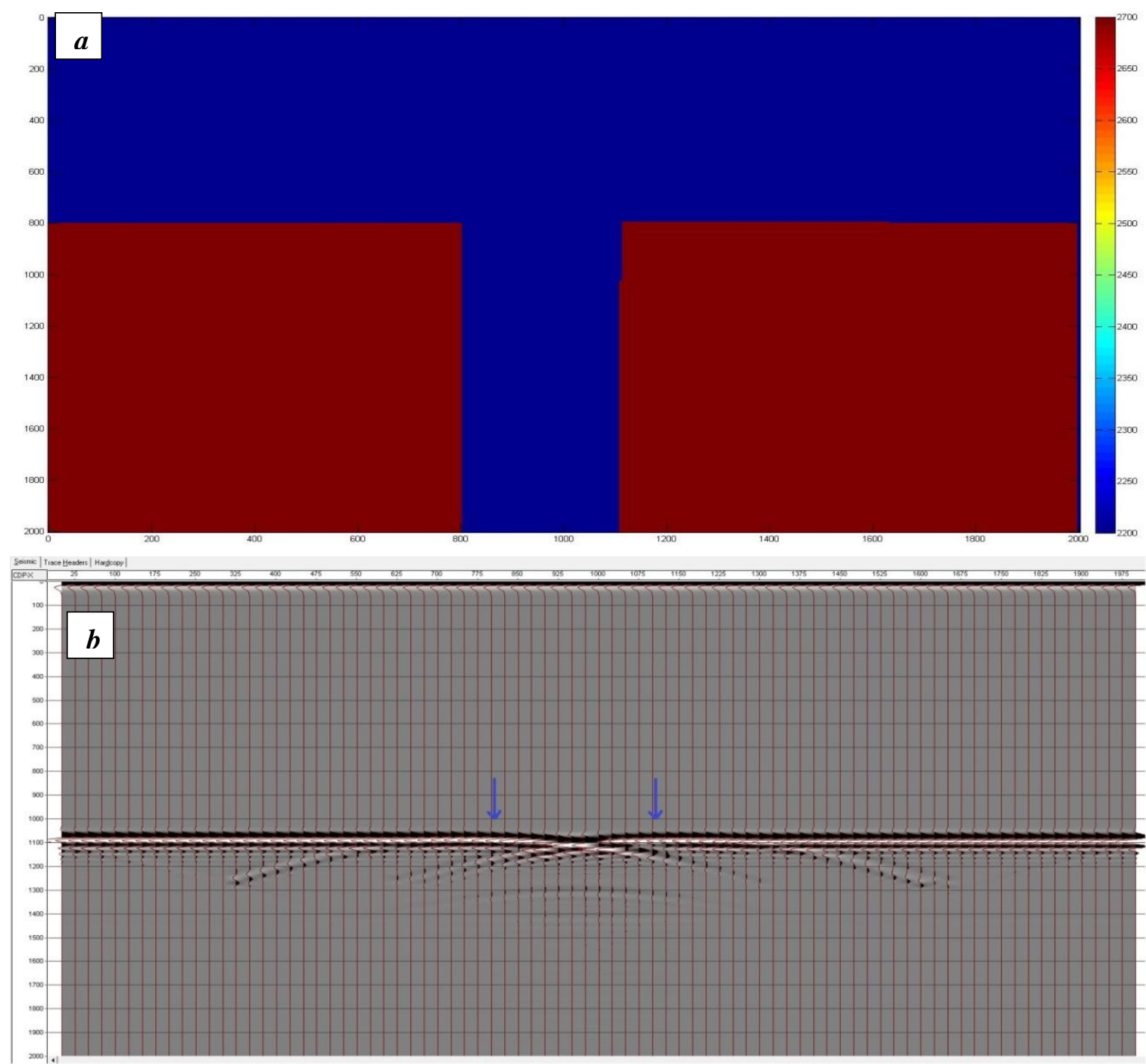

Figure.10: Two layer velocity model having different velocity $1500 \mathrm{~m} / \mathrm{sec}$ and $3000 \mathrm{~m} / \mathrm{sec}$ and intruded layer width is $300 \mathrm{~m}$ (above). Zero-Offset seismic gather on model (below).

\section{Frequency Dependent Imaging:}
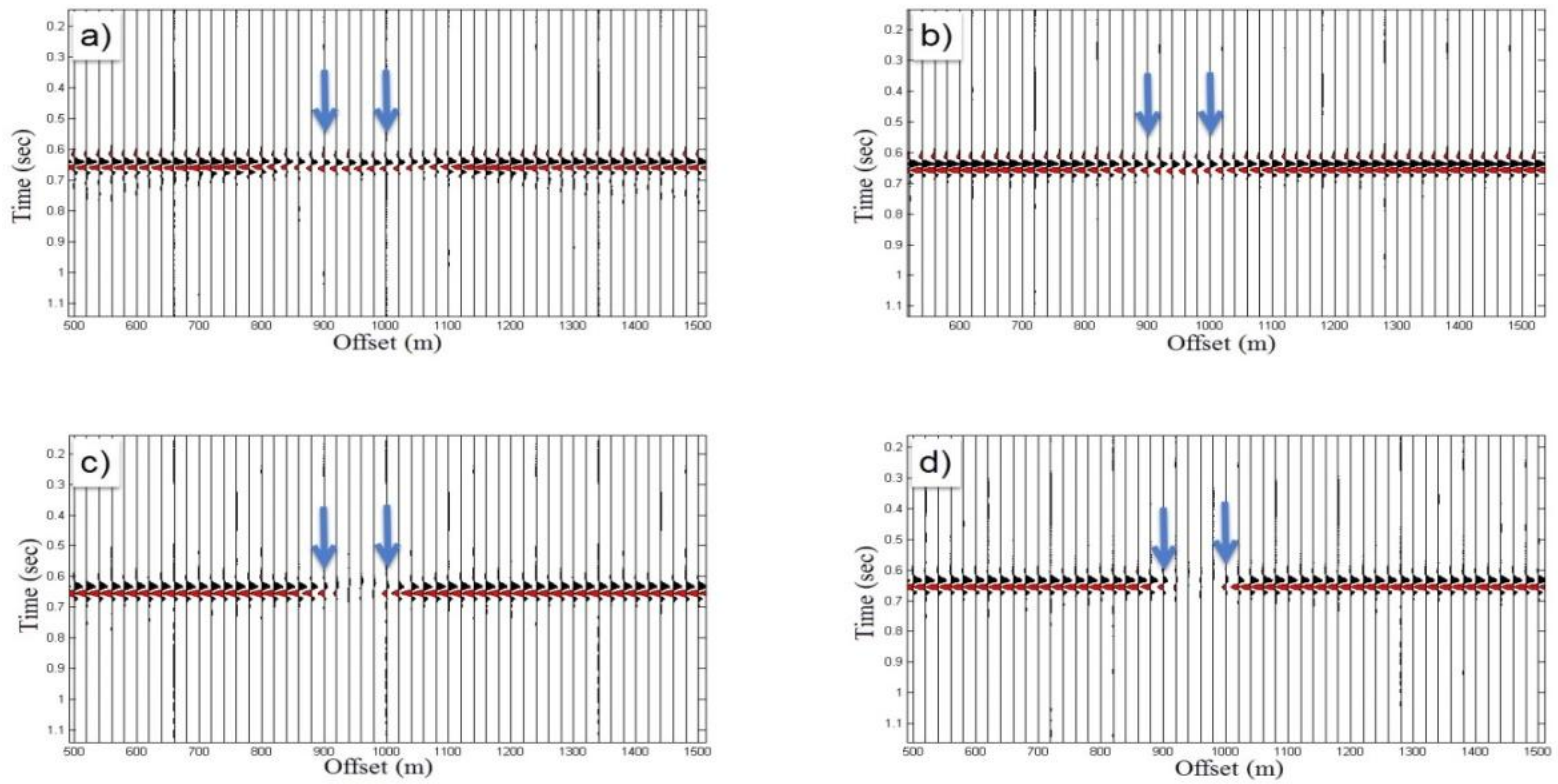

DOI: $10.9790 / 0990-0503015059$

Www.iosrjournals.org

$56 \mid$ Page 
Fig 11: Hole in reflection model-1 with a dominant frequency $30 \mathrm{~Hz}$ and a Hole width of 100m. (a) Zero-offset Synthetic Seismic gathers on Model-1, (b) (c) and (d) are traces after the application of the Kirchhoff Migration with different aperture sizes of 100, 300 and 500m, respectively.
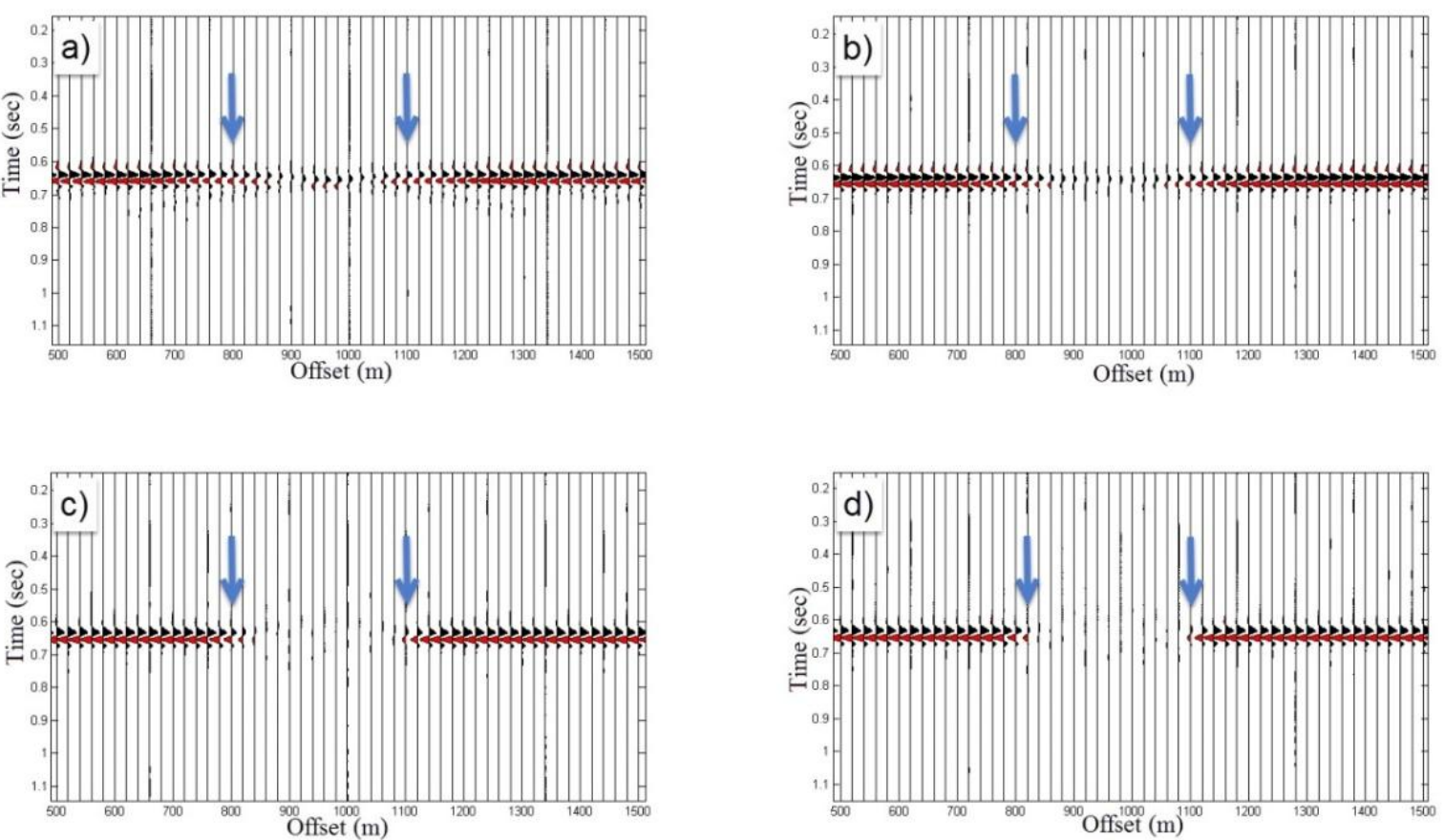

Fig. 12. Hole in reflection model with a dominant frequency of $30 \mathrm{~Hz}$ and Hole width of 300m. (a) Un-Image section, (b), (c) and (e) are migrated sections on different aperture sizes, 100, 300 and 500 m, respectively.
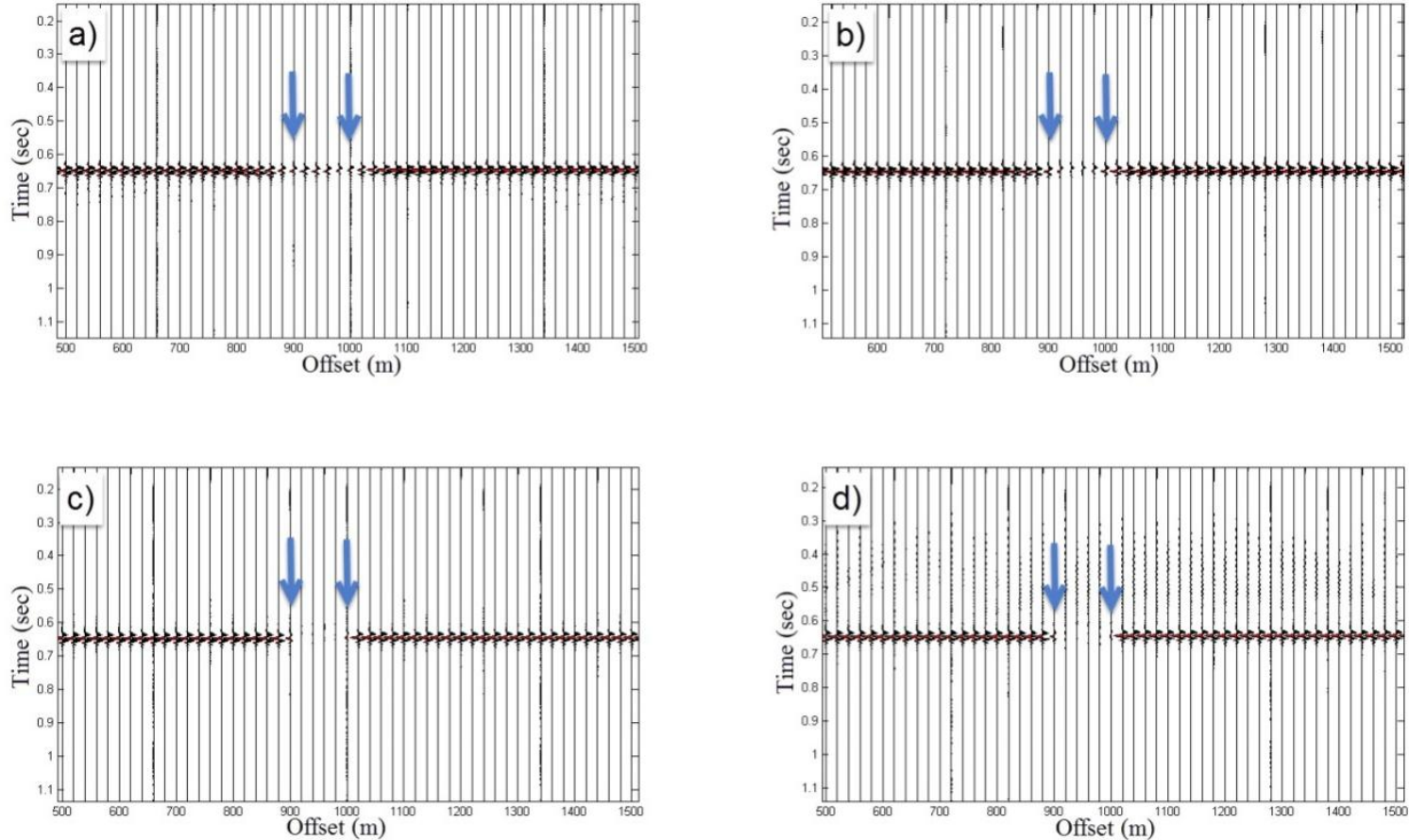

Fig 13: Shows traces with a dominant frequency of $80 \mathrm{~Hz}$ and a Hole width of 100m. (a) Zero-offset Synthetic Seismic gathers on Model-1, (b), (c) and (d) are traces after application of the Kirchhoff Migration with different aperture sizes. 

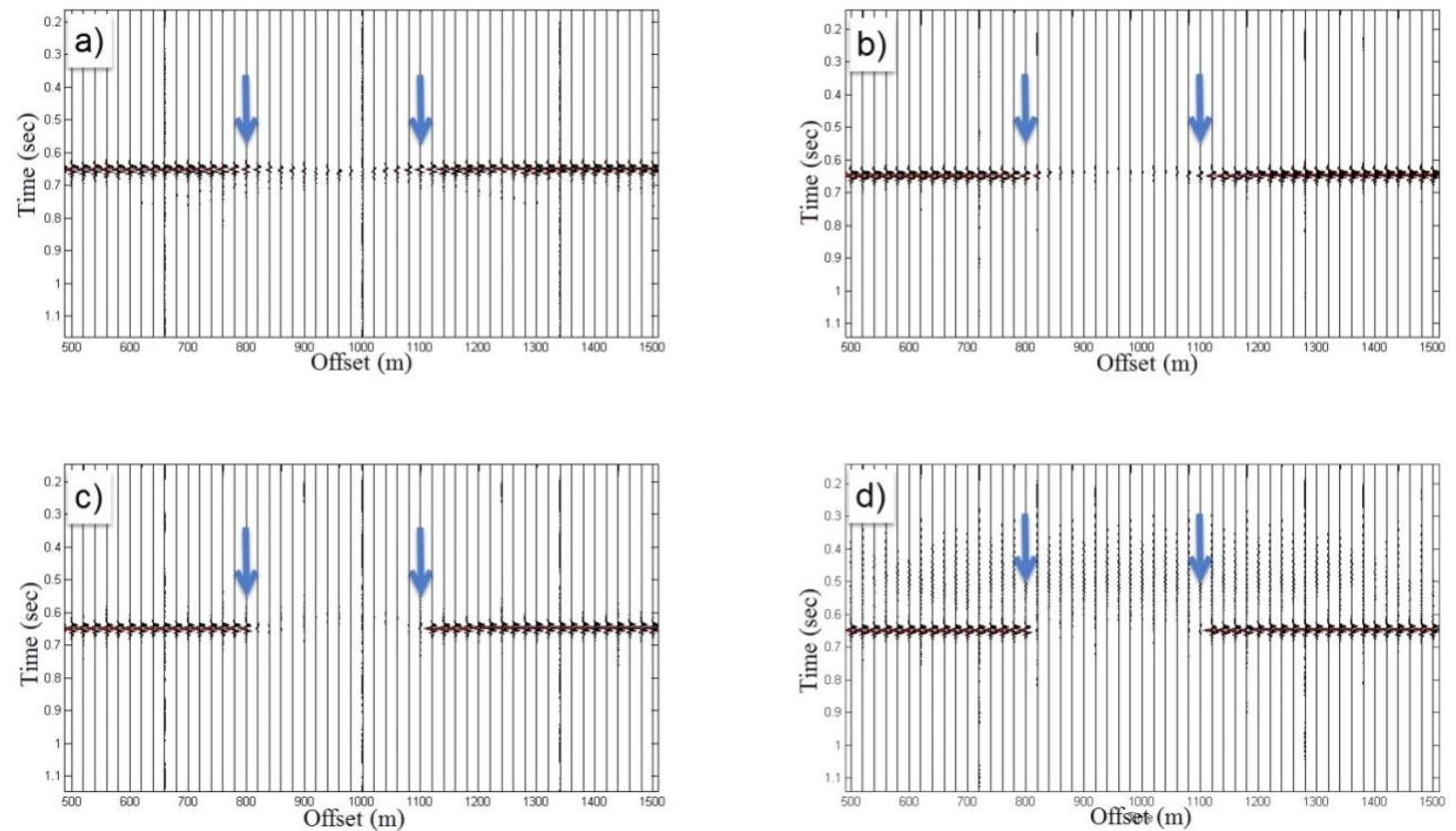

Fig 14: Hole in reflection synthetic gather with a dominant frequency $80 \mathrm{~Hz}$ and a Hole width of 300m. (a) Unimage section, (b) Migrated section with aperture of $100 \mathrm{~m}$, (c) Aperture of $300 \mathrm{~m}$ and (d) Aperture of $500 \mathrm{~m}$.

\section{Conclusions}

There are two major advantages of accurate seismic wave amplitude preservation. First, the correct positioning of the discontinuity such as fault, fracture and/or Karst, and secondly, the right amplitude recovery from the diffraction imaging is used for understanding of reservoir quality, thickness, net-to-gross, porosity and pore fill. In order to achieve this objective we should be able to understand the factors that affect the seismic amplitude and more importantly using correct imaging tools such as diffraction imaging to collapse the hyperbolic amplitude at the true position. In this paper, we implemented diffraction theory for explaining the diffraction hyperbolic behavior with different velocities and depth. Validation of diffraction theory is done by a synthetic earth model; Finite difference wave equation approximation is used for calculating synthetic seismic gathers for experiment. Implementation of double square root equation demonstrates that, the diffraction hyperbola is not dependent only on the velocity but also the depth.

High frequency seismic wave bounces less diffraction response than low-frequency wave and a $90^{\circ}$ phase change in amplitude of the diffraction hyperbola. Further, curvature of diffraction hyperbola is decrease as depth increase because of velocity increase. Dissimilar the reflection, the diffractions are always significantly affected by input velocity model for migration. They can be obtained with the application of the efficient migration-velocity analysis, which requires the shot gather data for accurately determination of velocity model for diffraction summation. This conclusion is reinforced when the analysis is repeated for the pitfall to avoid the wrong selection of migration aperture. Our outcome results demonstrate the high frequency data needs smaller size of migration aperture and low frequency data needs relatively larger migration aperture to preserve the diffraction amplitude for imaging as well as for hydrocarbon prediction. These diffraction summation and imaging analyses show the possibilities to save our computational power and cost of processing without improvement in data quality.

\section{Acknowledgments}

We are thankful to Universiti Teknologi PETRONAS (UTP) and the Centre of Excellence in Subsurface Seismic Imaging \& Hydrocarbon Prediction (CSI) to provide the facility for this research. Author also thankful to UTP\& PETRONAS staff for their valuable suggestions and comments. This work is supported by the sponsor Petroleum Research Fund (PRF) and some part of this work was presented in EAGE reservoir characterization workshop KL 2015. In this work, we have used Tesseral, MatLab and other tools like crewes, SegyMAT. 


\section{Reference}

[1] Bashir, Y., D. Ghosh, and C. W. Sum. "Detection of Fault and Fracture using Seismic Diffraction and Behavior of Diffraction Hyperbola with Velocity and Time." Seismic Driven Reservoir Characterization and Production Management. 2015.

[2] Bashir, Y., Ghosh, D. P., Alashloo, S. M., \& Sum, C. W. (2016). Effect of Frequency and Migration Aperture on Seismic Diffraction Imaging. In IOP Conference Series: Earth and Environmental Science (Vol. 30, No. 1, p. 012001). IOP Publishing.

[3] Berryhill, J. R. (1977). "DIFFRACTION RESPONSE FOR NONZERO SEPARATION OF SOURCE AND RECEIVER." GEOPHYSICS 42(6): 1158-1176.

[4] Ghosh., P. D. D. (2012). "Fundamental of Seismic Imaging With Examples From Asia Region." EAGE SLT Lecture Tour(2012).

[5] Hilterman, F. (1975). "Amplitudes of seismic waves; a quick look." GEOPHYSICS 40(5): 745-762.

[6] Hilterman, F. J. (1970). "THREE-DIMENSIONAL SEISMIC MODELING." GEOPHYSICS 35(6): 1020-1037.

[7] Landa, E. (2010). "Diffractions - Yesterday, Today, and Tomorrow." 72nd EAGE Conference \& Exhibition incorporating SPE EUROPEC 2010.

[8] Trorey, A. W. (1970). "A simple theory for seismic diffractions." GEOPHYSICS 35(5): 762-784.J.S. Bridle, "Probabilistic Interpretation of Feedforward Classification Network Outputs, with Relationships to Statistical Pattern Recognition," Neurocomputing-Algorithms, Architectures and Applications, F. Fogelman-Soulie and J. Herault, eds., NATO ASI Series F68, Berlin: Springer-Verlag, pp. 227-236, 1989. (Book style with paper title and editor) 